\title{
STRING DYNAMICS IN COSMOLOGICAL AND BLACK HOLE BACKGROUNDS: THE NULL STRING EXPANSION
}

\author{
C. O. LOUSTO \\ Department of Physics, University of Utah \\ Salt Lake City, UT 84112, USA \\ N. SÁNCHEZ \\ Observatoire de Paris, DEMIRM, UA 336 Laboratoire associé au CNRS, \\ 61, Avenue de l'Observatoire, F-75014 Paris, France
}

(June 22, 2018)

\begin{abstract}
We study the classical dynamics of a bosonic string in the $D$-dimensional flat Friedmann-Robertson-Walker and Schwarzschild backgrounds. We make a perturbative development in the string coordinates around a null string configuration; the background geometry is taken into account exactly. In the cosmological case we uncouple and solve the first order fluctuations; the string time evolution with the conformal gauge world-sheet $\tau$-coordinate is given by $X^{0}(\sigma, \tau)=q(\sigma) \tau^{\frac{1}{1+2 \beta}}+c^{2} B^{0}(\sigma, \tau)+\cdots, B^{0}(\sigma, \tau)=\sum_{k} b_{k}(\sigma) \tau^{k}$ where $b_{k}(\sigma)$ are given by Eqs. (3.15), and $\beta$ is the exponent of the conformal factor in the Friedmann-Robertson-Walker metric, i.e. $R \sim \eta^{\beta}$. The string proper size, at first order in the fluctuations, grows like the conformal factor $R(\eta)$ and the string energy-momentum tensor corresponds to that of a null fluid. For a string in the black hole background, we study the planar case, but keep the dimensionality of the spacetime $D$ generic. In the null string expansion, the radial, azimuthal, and time coordinates $(r, \phi, t)$ are $r=\sum_{n} A_{n}^{1}(\sigma)(-\tau)^{2 n /(D+1)}, \phi=\sum_{n} A_{n}^{3}(\sigma)(-\tau)^{(D-5+2 n) /(D+1)}$, and $t=\sum_{n} A_{n}^{0}(\sigma)(-\tau)^{1+2 n(D-3) /(D+1)}$. The first terms of the series represent a generic approach to the Schwarzschild singularity at $r=0$. First and higher order string perturbations contribute with higher powers of $\tau$. The integrated string energy-momentum tensor corresponds to that of a null fluid in $D-1$ dimensions. As the string approaches the $r=0$ singularity its proper size grows indefinitely like $\sim(-\tau)^{-(D-3) /(D+1)}$. We end the paper giving three particular exact string solutions inside the black hole. They represent respectively straight strings across the origin, twisted, and rigidly rotating strings.
\end{abstract}

11.25.Db, 04.70.Bw, 98.80.Hw

Typeset using REVTEX 


\section{INTRODUCTION}

The investigation of strings in curved spacetime is currently the best framework to study the physics of gravitation in the context of string theory. The study of string propagation in curved spacetimes provides new insights with respect to string theory in flat, Minkowski spacetime (and with respect to quantum fields in curved spacetime) [See for example Refs. [1 4.].] The results of this study apply to fundamental strings, as well as to cosmic strings, which behave essentially in a classical way.

The string equations of motion and constraints in curved spacetime are highly non linear and, in general, non exactly solvable. Different methods are available to solve this system: The string perturbation approach [5], the $\tau$-expansion method [6] (which provides exact local solutions for any background), the null string approach [7], and the construction of global solutions (by solitonic and inverse scattering methods, for instance), which allowed to uncover the new feature of multistring solutions [8 [1]. The expansion methods are described and classified by using the world sheet velocity of light, $c$, as an expansion parameter, in Sec. II below.

An approximate but general method is the expansion around the center of mass solution of the string (satisfying the geodesic equation of motion), the world sheet time $\tau$ being identified with the proper time associated to the center of mass trajectory. In this paper we are concerned with another general approximation method: The null string approach. In this approach, the string equations of motion and constraints are systematically expanded in powers of the parameter $c$ (the speed of light in the world sheet). This corresponds to a small string tension expansion. To zeroth order the string is effectively equivalent to a continuous line-beam of massless particles labeled by the world sheet spatial parameter $\sigma$. The points on the "null" string do not interact among them, but they interact with the gravitational background. Note the extended character of the zeroth order solution in the null string approach, as opposed to the perturbation approach in which the zeroth order is given by the string's center of mass point-like approximation

We also study fluctuations around the null string configuration, which naturally appears as an expansion in powers of $\tau$ with precisely defined $\sigma$-dependent coefficients. The string coordinates are expressed as

$$
X^{A}(\sigma, \tau)=A^{A}(\sigma, \tau)+c^{2} B^{A}(\sigma, \tau)+c^{4} C^{A}(\sigma, \tau)+\ldots
$$

the zeroth order $A^{A}(\sigma, \tau)$, first and second order fluctuations $B^{A}(\sigma, \tau), C^{A}(\sigma, \tau)$, satisfy Eqs. (2.3)-(2.4) as described in Sec. II. This null string approach is a powerful description for strings in the strong gravitational field regime, as is the case of black hole backgrounds near the $r=0$ singularity and in inflationary cosmological spacetimes, at late times, where string instabilities develop. The string perturbation method around the string center of mass allows to detect the emergence of instabilities [12,13,11], but is unable to describe the highly unstable regime [6]. We apply this null string expansion to cosmological spacetimes and to strings in the interior of black holes, falling into the singularity at $r=0$. In FriedmannRobertson-Walker cosmological spacetimes with conformal expansion factor $R \propto \eta^{\beta}, \eta$ being the conformal gauge world sheet time, the null string evolution can be expressed as

$$
X^{0}(\sigma, \tau)=q(\sigma) \tau^{\frac{1}{1+2 \beta}}+c^{2} B^{0}(\sigma, \tau)+\ldots
$$


where

$$
B^{0}(\sigma, \tau)=\sum_{k} b_{k}(\sigma) \tau^{k}, \quad k=2,2 \pm \alpha / \beta, k_{ \pm}
$$

The spatial coordinates $X^{i}(\sigma, \tau)$ are given by Eqs. (3.5) and (3.22) in Sec. III.

The proper size of the string grows proportional to the expansion factor $R(\eta)$ and the string energy momentum tensor corresponds to that of a null fluid.

In black hole spacetimes we find the null string expansion inside the Schwarzschild black hole. I is given by

$$
\begin{gathered}
t \doteq X^{0}(\sigma, \tau)=\sum_{n=1}^{\infty} A_{n}^{0}(\sigma)(-\tau)^{1+2 n(D-3) /(D+1)}+c^{2} B^{0}(\sigma, \tau)+\cdots \\
r \doteq X^{1}(\sigma, \tau)=\sum_{n=1}^{\infty} A_{n}^{1}(\sigma)(-\tau)^{2 n /(D+1)}+c^{2} B^{1}(\sigma, \tau)+\cdots \\
\phi \doteq X^{3}(\sigma, \tau)=\sum_{n=1}^{\infty} A_{n}^{3}(\sigma)(-\tau)^{(D-5+2 n) /(D+1)}+c^{2} B^{3}(\sigma, \tau)+\cdots
\end{gathered}
$$

and we have taken, for simplicity, the string in the equatorial plane, i. e.

$$
\theta^{i} \doteq A^{i}=\pi / 2 \text { for } i=2,4,5, \ldots, D-1 .
$$

The $\tau$-dependence exhibited above represents a generic behavior near the $r \rightarrow 0$ singularity $(\tau \rightarrow 0)$, and generalizes to $D$-dimensions the case recently analyzed in Ref. [14]. Higher order fluctuations $c^{2} B^{\mu}, c^{4} C^{\mu}$, and so on, contribute with increasing orders of $\tau$.

The proper string size grows indefinitely as the string approaches the $r \rightarrow 0$ singularity as

$$
\left(\frac{d l}{d \sigma}\right) \rightarrow\left(\frac{R_{S}}{r}\right)^{(D-3) / 2} X^{0^{\prime}}(\sigma, \tau=0) \rightarrow(-\tau)^{-(D-3) /(D+1)}
$$

The integrated string energy-momentum tensor for $r \rightarrow 0$ behaves as

$$
\Theta_{r}^{r} \rightarrow-\frac{L}{2 \pi \alpha^{\prime} r}=-\Theta_{\phi}^{\phi}, \quad \Theta_{t}^{t} \rightarrow 0 .
$$

$L$ being the string orbit angular momentum. In the infalling towards the $r \rightarrow 0$ singularity, the string behaves as a null fluid in $(D-1)$-dimensions; in particular, a planar string behaves as a two (spatially) dimensional null fluid. The emergence of string instabilities in Schwarzschild and Reissner-Nordström spacetimes, and the growing of the string proper size near the black hole singularity was first found in Ref. [13 using the perturbative expansion method around the string center of mass (although such expansion does not allow to describe the highly unstable regime and the full approach to the $r \rightarrow 0$ singularity).

This paper is organized as follows: In Sec. II we classify the different expansion methods to solve the string dynamics in curved spacetimes in terms of the parameter $c$ (or equivalently, in terms of the ratio between $\dot{X}^{A}$ and $c X^{A^{\prime}}$, the $\tau$ and $\sigma$ derivatives of the string coordinates), with particular focusing on the null string expansion (See also Table II). In Sec. III we solve the null string expansion in cosmological spacetimes. In Sec. IV we find the null string expansion near the Schwarzschild $r \rightarrow 0$ singularity. In Sec. $\mathrm{V}$ we find particular exact solutions inside the black hole event horizon describing straight string across $r=0$, twisted and rigidly rotating strings. Sec. VI summarizes our conclusions. 


\section{STRING DYNAMICS IN GRAVITATIONAL BACKGROUNDS}

The action of a bosonic string in a $D$-dimensional curved manifold endowed with a metric $G_{A B}(0 \leq A, B \leq D-1)$ is given by 15,16

$$
S=-T_{0} \int d \sigma d \tau \sqrt{-\operatorname{det} g_{\mu \nu}}
$$

where $g_{\mu \nu}=G_{A B}(X) \partial_{\mu} X^{A} \partial_{\nu} X^{B}$ is the two dimensional world-sheet metric $(0 \leq \mu, \nu \leq 1)$ and $T_{0}=1 /\left(2 \pi \alpha^{\prime}\right)$ is the string tension.

By use of the reparametrization invariance of the world-sheet one can take the conformal gauge, i.e. $g_{\mu \nu}=\rho(\sigma, \tau) \eta_{\mu \nu}$, where $\eta_{\mu \nu}$ is the two-dimensional Minkowskian metric. In this gauge, the classical equations of motion derived from the action (2.1) read

$$
\partial_{\tau}^{2} X^{A}-c^{2} \partial_{\sigma} X^{A}+\Gamma_{B C}^{A}\left[\partial_{\tau} X^{B} \partial_{\tau} X^{C}-c^{2} \partial_{\sigma} X^{B} \partial_{\sigma} X^{C}\right]=0
$$

where $\Gamma_{B C}^{A}$ are the Christoffel symbols associated to the metric $G_{A B}$ and we have introduced the velocity of wave propagation along the string (velocity of light), $c$, as the second fundamental constant after the string tension.

Variation of action (2.1) with respect to the world-sheet variables yields the two nontrivial constraints

$$
\begin{gathered}
\partial_{\tau} X^{A} \partial_{\sigma} X^{B} G_{A B}=0 \\
{\left[\partial_{\tau} X^{A} \partial_{\tau} X^{B}+c^{2} \partial_{\sigma} X^{A} \partial_{\sigma} X^{B}\right] G_{A B}=0 .}
\end{gathered}
$$

de Vega and Sánchez [5] have proposed a method to solve, both classically and quantum mechanically, the equations of motion and constraints of strings in curved spacetimes, i. e. Eqs. (2.2)-(2.4). This method treats the spacetime geometry exactly and considers the string excitations small as compared to the energy scales associated to the background gravitational metric. This method is particularly well suited to study strings in strong gravitational fields such us in black holes and in cosmological scenarios as opposed to the usual treatment in flat spacetime. The starting point is to consider a particular solution to the equations of motion and constraints, $X_{\text {part }}^{A}(\sigma, \tau)=A^{A}(\sigma, \tau)$ and then successively studying first, second and higher order fluctuations around this particular solution; to be denoted respectively as $B^{A}, C^{A}$, etc.

In Ref. [7] have been raised the interesting possibility of using $c$, the world-sheet light velocity, as the expansion parameter in the above development. This approach allow us to classify the different solutions proposed in the literature:

- i) $c \ll 1$ or equivalently, as can be seen from the field and constraint equations (2.2)(2.4), $\partial_{\tau} A^{A} \gg c \partial_{\sigma} A^{A}$ (holding also this inequality for second derivatives).

- ii) $c=1$ and thus all derivatives and other terms are, in principle, relevant in Eqs. (2.2)-(2.4).

- iii) $c \gg 1$ or equivalently, $c \partial_{\sigma} A^{A} \gg \partial_{\tau} A^{A}$ (holding also for second derivatives). 
- iv) There can be, of course, several other possibilities such as hybrid starting solutions that for some components of $A^{A}$ (and eventually $B^{A}, C^{A}$, etc) fulfill one of the cases i), ii) or iii) and for other components a different case.

More explicitly, we have

Case i) $c \ll 1$ : The appropriate development reads

$$
X^{A}(\sigma, \tau)=A^{A}(\sigma, \tau)+c^{2} B^{A}(\sigma, \tau)+c^{4} C^{A}(\sigma, \tau)+\ldots
$$

Plugging this into Eqs. (2.2)-(2.4) one obtains [7], to zeroth order in $c$ (since now on we adopt the notation ${ }^{\circ}=\partial_{\tau}$ and ${ }^{\prime}=\partial_{\sigma}$ )

$$
\begin{gathered}
\ddot{A}^{A}+\Gamma_{B C}^{A} \dot{A}^{B} \dot{A}^{C}=0, \\
\dot{A}^{B} \dot{A}^{C} G_{B C}=0, \\
\dot{A}^{B} A^{\prime C} G_{B C}=0 .
\end{gathered}
$$

Eq. (2.6) is the geodesic path followed by each point of the string. Eq. (2.7) means that this is a null geodesic and Eq. (2.8) equation states that the velocity is perpendicular to the string.

First order fluctuations around this particular solution $A(\sigma, \tau)$ can be obtained by retaining terms of order $c^{2}$ in Eqs (2.2)-(2.4)

$$
\begin{gathered}
\ddot{B}^{A}+2 \Gamma_{B C}^{A} \dot{A}^{B} \dot{B}^{C}+\Gamma_{B C, D}^{A} \dot{A}^{B} \dot{A}^{C} B^{D}=A^{\prime \prime A}+\Gamma_{B C}^{A} A^{\prime B} A^{\prime C}, \\
\left(2 \dot{A}^{B} \dot{B}^{C}+A^{\prime B} A^{\prime C}\right) G_{B C}+G_{B C, D} \dot{A}^{B} \dot{A}^{C} B^{D}=0, \\
\left(\dot{B}^{B} A^{\prime C}+\dot{A}^{B} B^{\prime C}\right) G_{B C}+G_{B C, D} \dot{A}^{B} A^{\prime C} B^{D}=0 .
\end{gathered}
$$

Higher order corrections can thus be systematically obtained. The interpretation of these equations is that they give the high energy (compared to the Planck energy) string behavior, corresponding to the limit of vanishing tension (and thus small $c$ ).

Case ii) $c=1$, and thus all derivatives and other terms are, in principle, relevant in Eqs. (2.2)-(2.4). One can thus make developments around the center-of-mass motion as a physically appealing starting solution. In fact, de Vega and Sánchez [5] have proposed in his original approach

$$
X^{A}=A^{A}(\sigma, \tau)+B^{A}(\sigma, \tau)+C^{A}(\sigma, \tau)+\ldots,
$$

where, $A^{A}(\tau)$ follows the geodesic equation

$$
\ddot{A}^{A}+\Gamma_{B C}^{A} \dot{A}^{B} \dot{A}^{C}=0,
$$

and the constraint 


$$
\dot{A}^{B} \dot{A}^{C} G_{B C}=0,
$$

means that these geodesics are null.

First order fluctuation equations now read

$$
\ddot{B}^{A}-B^{\prime \prime A}+2 \Gamma_{B C}^{A} \dot{A}^{B} \dot{B}^{C}+\Gamma_{B C, D}^{A} \dot{A}^{B} \dot{A}^{C} B^{D}=0,
$$

and

$$
\begin{gathered}
2 G_{B C} \dot{A}^{B} \dot{B}^{C}+G_{B C, D} \dot{A}^{B} \dot{A}^{C} B^{D}=0, \\
G_{B C} \dot{A}^{B} B^{\prime C}=0 .
\end{gathered}
$$

Higher order corrections can be in this way systematically considered. Still, a Fourier series decomposition of the first order fluctuations can be made [5], i. e. $B^{A}(\sigma, \tau)=$ $\Sigma_{n} \exp (i n \sigma) \eta_{n}^{A}(\tau)$. Also, a decomposition in purely left $A^{A}(\sigma+\tau)$ or right movers $A^{A}(\sigma-\tau)$ can be used as a starting exact solution.

Case iii) $c \gg 1$ or equivalently, $c \partial_{\sigma} A^{A} \gg \partial_{\tau} A^{A}$ (holding also for second derivatives). The appropriate development reads in this case

$$
X^{A}(\sigma, \tau)=A^{A}(\sigma, \tau)+\frac{1}{c^{2}} B^{A}(\sigma, \tau)+\frac{1}{c^{4}} C^{A}(\sigma, \tau)+\ldots
$$

Plugging this expression into Eqs. (2.2)-(2.4) yields a set of equations that is equivalent to (2.6)-(2.11) upon the substitutions $\tau \leftrightarrow \sigma$ and $c \rightarrow c^{-1}$. The interpretation of this approximation is now exactly the opposite to case i), i. e. here the zeroth order dependence on $\sigma$ overwhelms that on $\tau$ and thus represent very low energy strings, frozen at first approximation. Solutions to this case can be obtained from solutions to case i) by making the above mentioned substitutions. These represent, at zeroth order, stationary solutions as opposed to the dynamical ones. While solutions to case i) are appropriate to describe strings in strong gravitational fields, for instance, near black hole singularities, i.e. $R \rightarrow 0$, solutions to the case iii) are rather appropriate for strings far away from black holes, i.e. $R \rightarrow \infty$. We thus have an approximate duality here, represented by the transformation $\tau \leftrightarrow \sigma$ and $R \leftrightarrow R^{-1}$, which map the solutions to the cases i) and iii) into one another.

Case iv): This corresponds to an hybrid case. The circular string ansatz is an example of this situation:

$$
X^{0}=X^{0}(\tau), \quad R=R(\tau), \quad \psi=\psi(\sigma), \quad \theta=\pi / 2 .
$$

Clearly $X^{0}$ and $R$ coordinates follow criterion i) while $\psi$ follows case iii) and $\theta$ could be assigned to case ii). The first order fluctuations around this starting solution have been studied in Ref. 17,11] with regards to the stability analysis as the string approaches the black hole singularity. The stationary string ansatz have been studied in Ref. [18].

Finally, one can consider exact or asymptotic particular string solutions that deserve study in its own. Asymptotic solutions in a Friedmann-Robertson-Walker background representing highly unstable strings have been found [6]. Exact string and multistring solutions in de Sitter spacetime have been found by solitonic methods (a single world-sheet describing 
multiple different and independent strings) [19,8,9,11]. Ring solutions in black holes [10] have also been studied. For the sake of completeness it is also worth to remark that the propagation of strings in shock wave and conical spacetimes has been exactly solved [20.21].

Two physical interesting quantities can be computed: The string energy-momentum tensor integrated over a spatial volume completely enclosing the string [22], at fixed time $X^{0}$

$$
I^{A B}\left(X^{0}\right)=\int \sqrt{-G} T^{A B}(X) d^{D-1} \vec{X}
$$

where

$$
\sqrt{-G} T^{A B}(X)=\frac{1}{2 \pi \alpha^{\prime}} \int d \sigma d \tau\left[\dot{X}^{A} \dot{X}^{B}-X^{\prime A} X^{\prime B}\right] \delta^{(D)}[X-X(\sigma, \tau)],
$$

and the invariant string size $l$ [3]

$$
d l^{2}=-\dot{X}^{A} \dot{X}^{B} G_{A B}(X) d \sigma^{2} .
$$

[Note that the differential string size has the form of an effective mass $m_{\text {eff }}(\tau, \sigma)$ for the geodesic motion, and actually represents a projection from the target space onto the world sheet].

\section{NULL STRING DYNAMICS IN COSMOLOGICAL BACKGROUNDS}

Let us consider as a simple application of the formalism above the isotropic cosmological geometry given by the conformally flat Friedmann-Robertson-Walker metric

$$
d s^{2}=R^{2}\left(X^{0}\right)\left[-\left(d X^{0}\right)^{2}+\left(d X^{1}\right)^{2}+\left(d X^{2}\right)^{2}+\cdots+\left(d X^{D-1}\right)^{2}\right],
$$

where we parametrize the conformal factor as $R\left(X^{0}\right)=K\left(X^{0} / \beta\right)^{\beta}$.

We are interested in this paper in the null string dynamics and the fluctuations around it. Zeroth order: The equations of motion (2.6) have the following first integral of motion (conservation of the four-moment $P_{A}$ )

$$
R^{2}\left(A^{0}\right) \dot{A}^{0}(\sigma, \tau)=-P_{0}(\sigma), \quad R^{2}\left(A^{0}\right) \dot{A}^{i}(\sigma, \tau)=P_{i}(\sigma) .
$$

where from now on $i, j$ run from 1 to $D-1$.

The constraints at $\tau=0$ yield

$$
\begin{gathered}
\left(P_{0}\right)^{2}=\sum_{i=1}^{D-1}\left(P_{i}\right)^{2}, \\
-P^{0}\left(\bar{A}^{0}\right)^{\prime}=\sum_{i=1}^{D-1} P^{i}\left(\bar{A}^{i}\right)^{\prime} .
\end{gathered}
$$

where we use the notation $A^{A}(\sigma, \tau=0)=\bar{A}^{A}$ 
From Eq. (3.2) we see that the only relevant $\tau$-dependence is that of $A^{0}(\tau)$. In fact, we can write

$$
A^{i}(\tau)-\bar{A}^{i}=\frac{P^{i}}{-P^{0}}\left[A^{0}(\tau)-\bar{A}^{0}\right] .
$$

By direct integration of the zeroth component of Eq. (3.2) one obtains

$$
A^{0}(\tau)-\bar{A}^{0}=\left[\frac{(1+2 \beta) \beta^{2 \beta} P^{0}}{K^{2}} \tau\right]^{\frac{1}{1+2 \beta}} \doteq q(\sigma) \tau^{\frac{1}{1+2 \beta}},
$$

The particular time-dependence of the scale factor as usually referred to in the literature is recalled in Table [. The cases dealt with in Refs. [7] are in $D=4$ and $\beta=-1 / 2$ and -1 respectively. Note that the cosmic time $t$ is related to the conformal time $X^{0} \approx A^{0}$ by

$$
t-t_{0}=\frac{K\left(A^{0}-\bar{A}^{0}\right)^{1+\beta}}{\beta^{\beta}(1+\beta)} .
$$

Thus, $\tau$ does not coincides with $t$.

We can now compute the zeroth order energy-momentum density tensor from Eq. (2.20)

$$
I_{A}^{B}(\sigma)=\frac{P_{A} P_{C} \eta^{C B}}{-P_{0} \alpha^{\prime}} .
$$

Hence, at zeroth order, the string energy and momentum keep constant as the universe evolves. The trace of $I_{A}^{B}$ vanishes to this zeroth order. Since, to this order, each point of the string follows a null geodesic, the proper size Eq. (2.22) also vanishes.

First order fluctuations: The 0 component of Eq. (2.9) yields

$$
\begin{gathered}
\ddot{B}^{0}+\frac{2 d R}{R d X^{0}}\left[\dot{A}^{0} \dot{B}^{0}+\dot{A}^{i} \dot{B}^{i}\right]+\frac{d}{d X^{0}}\left(\frac{d R}{R d X^{0}}\right)\left[\left(\dot{A}^{0}\right)^{2}+\left(\dot{A}^{i}\right)^{2}\right] B^{0}= \\
A^{0^{\prime \prime}}+\frac{d R}{R d X^{0}}\left[\left(A^{0^{\prime}}\right)^{2}+\left(A^{i^{\prime}}\right)^{2}\right] .
\end{gathered}
$$

With the help of the constraint equation (2.10)

$$
\left(2 \dot{B}^{A} A^{B^{\prime}}+\dot{A}^{A} B^{B^{\prime}}\right) \eta_{A B}=0
$$

we obtain an uncoupled equation for $B^{0}$

$$
\ddot{B}^{0}+\frac{4 \alpha}{\tau} \dot{B}^{0}-\frac{2 \alpha^{2}}{\beta \tau^{2}} B^{0}=A^{0^{\prime \prime}}+\frac{2\left(A^{i^{\prime}}\right)^{2}}{A^{0}} \doteq F^{0}(\sigma, \tau),
$$

where $\alpha=\beta /(\beta+1)$, and we have used that

$$
\frac{d R}{R d X^{0}}=\frac{\beta}{X^{0}}, \frac{d}{d X^{0}}\left(\frac{d R}{R d X^{0}}\right)=\frac{-\beta}{\left(X^{0}\right)^{2}} .
$$

We can write the general solution to Eq. (3.10) as 


$$
B^{0}(\sigma, \tau)=\sum_{k} b_{k}(\sigma) \tau^{k}
$$

where $b_{k_{+}}$and $b_{k_{-}}$are arbitrary constants corresponding to the general solution to the homogeneous equation associated to Eq. (3.10) $; k_{ \pm}=-(4 \alpha-1) / 2 \pm \sqrt{(2 \alpha-1 / 2)^{2}+2 \alpha^{2} / \beta}$. The other powers of $k$ correspond to the series development of the function $F^{0}(\sigma, \tau)$ and give the particular solution to the inhomogeneous equation.

To provide a simpler solvable example we take $\bar{A}^{0}=0$. In this case $F^{0}$ takes the following form:

$$
F^{0}(\sigma, \tau)=\left[q^{\prime \prime}+\frac{2 \beta}{q}\left(\frac{P_{i} q}{P_{0}}\right)^{\prime 2}\right] \tau^{\alpha / \beta}-\frac{4 \beta \bar{A}^{i^{\prime}}}{q}\left(\frac{P_{i} q}{P_{0}}\right)^{\prime}+\frac{\beta\left(\bar{A}^{i^{\prime}}\right)^{2}}{q} \tau^{-\alpha / \beta}
$$

Plugging this into the right hand side of Eq. (3.10) and using the form of $B^{0}$ given by Eq. (3.12) we obtain

$$
\sum_{k} b_{k}\left[k(k-1)+4 k \alpha-2 \alpha^{2} / \beta\right] \tau^{k-2}=F^{0}(\sigma, \tau)
$$

Thus, there are only three values of $k$ given by $k-2=0, \pm \alpha / \beta$ with the corresponding $b_{k}$ as defined by Eqs. (3.13) and (3.14)

$$
\begin{aligned}
b_{2} & =-\frac{4 \beta \bar{A}^{i^{\prime}}}{q\left[2+8 \alpha-2 \alpha^{2} / \beta\right]} \\
b_{2+\alpha / \beta} & =\frac{q^{\prime \prime}+\frac{2 \beta}{q}\left(\frac{P_{i} q}{P_{0}}\right)^{\prime 2}}{(2+\alpha / \beta)(1+4 \alpha+\alpha / \beta)-2 \alpha^{2} / \beta} \\
b_{2-\alpha / \beta} & =\frac{\beta\left(\bar{A}^{i^{\prime}}\right)^{2}}{q\left[(2-\alpha / \beta)(1+4 \alpha-\alpha / \beta)-2 \alpha^{2} / \beta\right]}
\end{aligned}
$$

Let us now consider the $i$ components of Eq. (2.9) for the first order fluctuations:

$$
\ddot{B}^{i}+\frac{2}{R} \frac{d R}{d X^{0}}\left[\dot{A}^{0} \dot{B}^{i}+\dot{A}^{i} \dot{B}^{0}\right]+2 \frac{d}{d X^{0}}\left(\frac{d R}{R d X^{0}}\right) \dot{A}^{0} \dot{A}^{i} B^{0}=A^{i^{\prime \prime}}+2 \frac{d R}{R d X^{0}} A^{0^{\prime}} A^{i^{\prime}} .
$$

With the help of Eq. (3.11)

$$
\ddot{B}^{i}+\frac{2 \alpha}{\tau} \dot{B}^{i}=A^{i^{\prime \prime}}+\frac{2 \beta A^{i^{\prime}} A^{0^{\prime}}}{A^{0}}+\frac{2 \alpha P_{i}}{P_{0} \tau}\left(\dot{B}^{0}-\frac{\alpha B^{0}}{\beta \tau}\right) \doteq F^{i}(\sigma, \tau),
$$

Known $B^{0}(\sigma, \tau)$ the general solution to this equation can be written in terms of quadratures

$$
B^{i}(\sigma, \tau)=\bar{B}^{i}+\overline{\dot{B}}^{i} \frac{\tau^{1-2 \alpha}}{1-2 \alpha}+\int^{\tau} d \tilde{\tilde{\tau}} \tilde{\tilde{\tau}}^{-2 \alpha} \int^{\tilde{\tilde{\tau}}} d \tilde{\tau} \tilde{\tau}^{2 \alpha} F^{i}(\sigma, \tilde{\tau})
$$

where $\bar{B}^{i}=B^{i}(\sigma, 0)$. Note that $\bar{B}^{i}$ and $\dot{\bar{B}}^{i}$ can be absorbed into $\bar{A}^{i}$ and $P_{i}$ respectively. We are thus left with the two constants $b_{k_{ \pm}}$which are fixed by the two first order constraints equations (2.10) and (2.11) 


$$
\begin{gathered}
\left(\dot{A}^{A} \dot{B}^{B}+A^{A^{\prime}} A^{B^{\prime}}\right) \eta_{A B}=0 \\
\left(2 \dot{B}^{A} A^{B^{\prime}}+\dot{A}^{A} B^{B^{\prime}}\right) \eta_{A B}=0 .
\end{gathered}
$$

We are thus sure we are not adding any spurious degree of freedom at first order.

We can again show the explicit dependence for the case $\bar{A}^{0}=0$. In this case $F^{i}$ takes the following form

$$
\begin{gathered}
F^{i}(\sigma, \tau)=\bar{A}^{i^{\prime \prime}}+2 b_{2} \alpha \frac{P_{i}}{P_{0}}(2-\alpha / \beta)+2 \beta \frac{q^{\prime}}{q} \bar{A}^{i^{\prime}}- \\
{\left[\left(\frac{P_{i} q}{P_{0}}\right)^{\prime \prime}+2 \beta \frac{q^{\prime}}{q}\left(\frac{P_{i} q}{P_{0}}\right)^{\prime}-4 \alpha b_{2+\alpha / \beta} \frac{P_{i}}{P_{0}}\right] \tau^{\alpha / \beta}+} \\
4 \alpha b_{2-\alpha / \beta} \frac{P_{i}}{P_{0}}(1-\alpha / \beta) \tau^{-\alpha / \beta}+2 \alpha b_{k_{ \pm}} \frac{P_{i}}{P_{0}}\left(\left(k_{ \pm}\right)-\alpha / \beta\right) \tau^{k_{ \pm}-2}
\end{gathered}
$$

or in a more compact way

$$
F^{i}(\sigma, \tau)=\sum_{n} F_{n}^{i}(\sigma) \tau^{n}, \quad n=0, \pm \alpha / \beta,\left(k_{ \pm}\right)-2
$$

where the notation $F_{n}^{i}$ clearly refers to the coefficient of the $n$-th power of $\tau$ in Eq. (3.20).

Plugging this into the right hand side of Eq. (3.18) and using the form of $B^{0}$ given by Eqs. (3.12) and (3.15), we obtain

$$
B^{i}(\sigma, \tau)=\bar{B}^{i}+\overline{\dot{B}}^{i} \frac{\tau^{1-2 \alpha}}{1-2 \alpha}+\sum_{n} \frac{F_{n}^{i} \tau^{n+2}}{(n+2)(2 \alpha+n+1)} \quad, \quad n=0, \pm \alpha / \beta,\left(k_{ \pm}\right)-2 .
$$

The proper string size that vanished to zeroth order now takes the following form

$$
d l^{2}=R^{2} \eta_{A B} A^{A^{\prime}} A^{B^{\prime}} d \sigma^{2}
$$

where we have used the constraint (3.19). We see that the string size grows like the conformal factor of the Friedmann-Robertson-Walker metric. This unstable behavior was already observed in Ref. [12] in the fluctuations around the center of mass zeroth order solution.

The coordinate fluctuations $B^{0}$ and $B^{i}$ evolve with several powers of $\tau$ and for a given value of the conformal factor exponent $\alpha$, we will have several regimes. We can see from the above expressions (Eq. (3.22) ) that in the regime of $R$ large, that is $\tau \rightarrow \infty$, for $\alpha / \beta>0$, i.e. $\beta>-1$, the overwhelming power will be $(2+\alpha / \beta)$ or $\left(2+k_{+}\right)$. When $\alpha / \beta<0$, i.e. $\beta<-1$, the regime of $R$ large is reached when $\tau \rightarrow 0$, then the predominant power will be $2+\alpha / \beta$ or $2+k_{-}$. Note also that these results do not depend explicitly on the dimension, $D$, since the metric coefficients neither do.

\section{BLACK HOLE SPACETIMES}

We are particularly interested in studying the dynamics of strings in black hole spacetimes and its approach to the singularity at radial coordinate $r=0$.

Let us consider a $D$-dimensional black hole background [23],

$$
d s^{2}=-g(r)\left(d X^{0}\right)^{2}+g(r)^{-1} d r^{2}+r^{2} d \Omega_{D-2},
$$


where for the Reissner-Nordström metric

$$
g(r)=1-\left(\frac{R_{S}}{r}\right)^{D-3}+\left(\frac{\tilde{Q}}{r}\right)^{2(D-3)} \quad, \quad \tilde{Q}^{2(D-3)}=\frac{8 \pi G Q^{2}}{(D-2)(D-3)}
$$

with $M$ and $Q$ being respectively the mass and charge of the black hole.

We can now study the string dynamics by using the null string approach described in Sec. II.

We then propose the following development for the string coordinates

$$
X^{A}(\sigma, \tau)=A^{A}(\sigma, \tau)+c^{2} B^{A}(\sigma, \tau)+\ldots
$$

Replacing this into the field equations (2.2), with (4.1) acting as the curved background, we find to zeroth order the following first integrals of motion

$$
\dot{\theta} \simeq \dot{A}^{2}=0, \quad \dot{\phi} \simeq A^{3}=\frac{L(\sigma)}{r^{2}}
$$

where for simplicity we consider a string lying on the equatorial plane. $L(\sigma)$ is the angular momentum of each point forming the string. The other first integrals of motion are

$$
\dot{X}^{0} \simeq \dot{A}^{0}=E(\sigma) g^{-1}(r), \quad \dot{r}^{2} \simeq\left(\dot{A}^{1}\right)^{2}=E^{2}(\sigma)-\frac{L^{2}(\sigma)}{r^{2}} g(r) .
$$

From this last equation one can see that for the Reissner-Nordström black hole, if $L(\sigma) \neq 0$, the string will only skirt the singularity. Since we are interested in the string approach to the singularity, we consider from now on Schwarzschild black holes (in $D$ dimensions).

The remaining constraint equation (2.8) at $\tau=0$ takes the following simple form

$$
\bar{A}^{0^{\prime}}(\sigma)=\frac{L(\sigma)}{E(\sigma)} \bar{A}^{3^{\prime}}(\sigma) .
$$

We can further integrate expression (4.5) near the black hole singularity to obtain

$$
A^{1}(\sigma, \tau) \simeq p(\sigma)(-\tau)^{\frac{2}{D+1}}, \quad p(\sigma)=\left[\frac{1}{2}(D+1) R_{S}^{\frac{D-3}{2}} L(\sigma)\right]^{\frac{2}{D+1}}
$$

where we have chosen $\tau \leq 0$ such that $A^{1}(\tau=0)=0 . \tau$ measures the proper time of infall to the singularity at $r=0$.

By use of the above expression we find

$$
A^{0}(\sigma, \tau) \simeq \bar{A}^{0}+\frac{(D+1)}{(3 D-5)} E\left(\frac{p(\sigma)}{R_{S}}\right)^{D-3}(-\tau)^{\frac{3 D-5}{D+1}}
$$

and

$$
\begin{gathered}
A^{3}(\sigma, \tau) \simeq \bar{A}^{3}-\frac{(D+1)}{(D-3)} \frac{L(\sigma)}{p^{2}(\sigma)}(-\tau)^{\frac{D-3}{D+1}} \\
A^{i}=\pi / 2 \text { for } i=2,4,5, \ldots, D-1
\end{gathered}
$$


Comparison of these expressions with the corresponding ones found in Ref [13], (Eq. (88)), shows that they have the same $\tau$ dependence. The difference lies in the prefactors: In Eqs. (4.7)-(4.8) they are $\sigma$-dependent while in those of Ref [13] they are constant. This is due to the different zeroth order solution we have chosen. In Ref. [13] we have taken the center of mass motion which is a point-like solution, while now we have a null string, an extended zeroth order solution.

We can consider the approach to the singularity with higher powers of $A^{1}$ (our radial coordinate). This yields the following power dependence in $\tau$

$$
A^{1}=\sum_{n=1}^{\infty} A_{n}^{1}(\sigma)(-\tau)^{2 n /(D+1)},
$$

and then

$$
\begin{aligned}
& A^{3}=\sum_{n=1}^{\infty} A_{n}^{3}(\sigma)(-\tau)^{(D-5+2 n) /(D+1)}, \\
& A^{0}=\sum_{n=1}^{\infty} A_{n}^{0}(\sigma)(-\tau)^{1+2 n(D-3) /(D+1)} .
\end{aligned}
$$

This $\tau$ dependence seems to be a quite general behavior near singularities and generalize to $D$-dimensions the case analyzed recently in Ref ( $[14])$. There will still be higher order corrections coming form the first order fluctuations $B^{\mu}$, second order fluctuations $C^{\mu}$, and so on. We have already seen that in the cosmological case, five different powers of $\tau$ contribute to the first order.

The proper string size that at zeroth order vanished for null string, does not vanish at higher order. In order to compute the first order correction to the string size we do not need to explicitly compute the $B^{A}$ terms. In fact, by use of the constraint (2.4), Eq. (2.22) can be rewritten as

$$
\left(\frac{d l}{d \sigma}\right)^{2}=X^{A^{\prime}} X^{B^{\prime}} G_{A B}(X) .
$$

Plugging expressions (4.5)-(4.4) into this equation and making use of the constraint (2.8) to eliminate $A^{1^{\prime}}$, we obtain

$$
\left(\frac{d l}{d \sigma}\right)^{2}=-g\left(A^{0^{\prime}}\right)^{2}+\left(A^{1}\right)^{2}\left(A^{3^{\prime}}\right)^{2}+g \frac{\left(E A^{0^{\prime}}-L A^{3^{\prime}}\right)^{2}}{\left(E^{2}-L^{2} g /\left(A^{1}\right)^{2}\right)} .
$$

As the string approachs the singularity it diverges in the following way

$$
\left(\frac{d l}{d \sigma}\right)^{2} \rightarrow\left(\frac{R_{S}}{r}\right)^{D-3}\left(\bar{X}^{0^{\prime}}\right)^{2} \rightarrow(-\tau)^{-2(D-3) /(D+1)} .
$$

This unstable behavior have already been discovered in Ref [13], where we studied string propagation in $D$-dimensional Reissner-Nordström black holes under the approximation labeled by $c=1$. We solved there the first order string fluctuations around the center of 
mass motion at spatial infinity, near the event horizon and at the spacetime singularity. In Reissner-Nordström black holes the radial components and angular string components develop instabilities as the string approachs the singularity. The string motion is like that of a particle in a strongly attractive potential proportional to $-(\Delta \tau)^{-2}$. As $\Delta \tau \rightarrow 0$ the string ends trapped by the black hole singularity.

We can also compute the integrated energy-momentum tensor from expressions (2.20) and (2.21). Using Eqs. (4.5)-(4.4) we find

$$
\Theta^{A B}=\frac{d I^{A B}}{d \sigma} \approx \frac{1}{2 \pi \alpha^{\prime}} \frac{\sqrt{-g} \dot{A}^{A} \dot{A}^{B}}{\sqrt{E^{2}-L^{2} g /\left(A^{1}\right)^{2}}},
$$

where we have taken into account that inside the black hole $A^{0}$ is spacelike and $A^{1}$ is timelike.

In the limit $r \rightarrow 0$, we have

$$
\Theta_{1}^{1} \rightarrow-\frac{L}{2 \pi \alpha^{\prime} r}=-\Theta_{3}^{3}, \quad \Theta_{0}^{0} \rightarrow 0
$$

We first observe that the results are independent of the spacetime dimensionality, $D$. The facts that the trace vanishes (since we are dealing with a null system), and that $\Theta_{0}^{0} \rightarrow 0$, reduce the system to a $D-1$-dimensional null system (in our example of a planar string, this reduces the system to a two dimensional null string).

\section{EXACT STRING SOLUTIONS INSIDE A BLACK HOLE}

In four dimensions the equations of motion of a string in the curved background (4.1) read 18

$$
\begin{aligned}
& \ddot{t}-t^{\prime \prime}+\frac{g_{, r}}{g}\left(\dot{t} \dot{r}-t^{\prime} r^{\prime}\right)=0, \\
& \ddot{r}-r^{\prime \prime}-\frac{g_{, r}}{2 g}\left(\dot{r}^{2}-r^{2}\right)+\frac{g g, r}{2}\left(\dot{t}^{2}-t^{2}\right)-g r\left(\dot{\theta}^{2}-\theta^{2}\right)-g r \sin ^{2} \theta\left(\dot{\phi}^{2}-\phi^{2}\right)=0, \\
& \ddot{\theta}-\theta^{\prime \prime}+\frac{2}{r}\left(\dot{\theta} \dot{r}-\theta^{\prime} r^{\prime}\right)-\sin \theta \cos \theta\left(\dot{\phi}^{2}-\phi^{\prime 2}\right)=0, \\
& \ddot{\phi}-\phi^{\prime \prime}+\frac{2}{r}\left(\dot{\phi} \dot{r}-\phi^{\prime} r^{\prime}\right)+2 \cot \theta\left(\dot{\theta} \dot{\phi}-\theta^{\prime} \phi^{\prime}\right)=0,
\end{aligned}
$$

and the constraints

$$
\begin{aligned}
& -g \dot{t} t^{\prime}+\frac{1}{g} \dot{r} r^{\prime}+r^{2} \dot{\theta} \theta^{\prime}+r^{2} \sin ^{2} \theta \dot{\phi} \phi^{\prime}=0, \\
& -g\left(\dot{t}^{2}+t^{\prime 2}\right)+\frac{1}{g}\left(\dot{r}^{2}+r^{2}\right)+r^{2}\left(\dot{\theta}^{2}+\theta^{\prime 2}\right)+r^{2} \sin ^{2} \theta\left(\dot{\phi}^{2}+\phi^{2}\right)=0 .
\end{aligned}
$$

We are interested in finding solutions inside the black hole, where we know that the $r$ coordinate becomes timelike while the $t$ coordinate is spacelike. We thus look for solutions of the following particular form

$$
r=r(\tau) \doteq \mathcal{T}(\tau) \quad \text { and } \quad t=t(\sigma) \doteq \mathcal{R}(\sigma)
$$


Using this ansatz the first equation of motion in (5.1) give us

$$
\mathcal{R}(\sigma)=E \sigma+t_{0},
$$

where $E$ and $t_{0}$ are arbitrary constants.

From the first constraint Eq. (5.2) we have

$$
\mathcal{T}(\tau)^{2} \sin ^{2} \theta \dot{\phi} \phi^{\prime}=0 .
$$

In terms of Kruskal coordinates we have

$$
\begin{aligned}
& u(\sigma, \tau)=\left(1-\frac{\mathcal{T}(\tau)}{2 M}\right)^{1 / 2} e^{\frac{\mathcal{T}(\tau)}{4 M}} \sinh \left(\frac{\mathcal{R}(\sigma)}{4 M}\right) \\
& v(\sigma, \tau)=\left(1-\frac{\mathcal{T}(\tau)}{2 M}\right)^{1 / 2} e^{\frac{\mathcal{T}(\tau)}{4 M}} \cosh \left(\frac{\mathcal{R}(\sigma)}{4 M}\right)
\end{aligned}
$$

Eq. (5.5) allows us to analyze three cases:

a) $\theta=$ constant $\neq \pi / 2$. Then $\phi$ is also constant and

$$
\dot{\mathcal{T}}(\tau)= \pm E g(\mathcal{T})
$$

represents a straight string in the two dimensional $\mathcal{R}-\phi$ space.

b) For $\theta=\pi / 2$, i.e. the planar case, we have:

b1) $\dot{\phi}=0$. Then, from the equation of motion of $\phi$ we deduce $\phi=n \sigma+\phi_{0}$, where $n$ and $\phi_{0}$ are arbitrary constants. While for the timelike coordinate $\mathcal{T}(\tau)$ we have

$$
\dot{\mathcal{T}}(\tau)^{2}=E^{2} g(\mathcal{T})^{2}-n^{2} \mathcal{T}(\tau)^{2} .
$$

This solution represents a straight twisted string in the $\mathcal{R}-\phi$ space.

b2) $\phi^{\prime}=0$. Then, from the equation of motion of $\phi$ we deduce $\dot{\phi}=L / \mathcal{T}(\tau)^{2}$. Here $L$ is a constant and now

$$
\dot{\mathcal{T}}(\tau)^{2}=E^{2} g(\mathcal{T})^{2}-g(\mathcal{T}) \frac{L^{2}}{\mathcal{T}(\tau)^{2}} .
$$

This solution represents a rigid rotating straight string in the $\mathcal{R}-\phi$ space.

\section{CONCLUSION}

We have performed a systematic study of the null string expansion method to solve the string equations of motion and constraints in curved spacetimes. This corresponds

to a small string tension expansion (inverse powers of $\alpha^{\prime}$ ). The perturbative expansion series is conveniently described in terms of the parameter $c$, the wave propagation velocity along the string. The zeroth order describes a null string configuration; first and higher order fluctuations around it are systematically constructed. The null string expansion series in $D$-dimensional Friedmann-Robertson-Walker and Schwarzschild spacetimes have been computed. In the latter case, the first terms of the series represent a generic approach to 
the string falling towards the $r=0$ singularity. The string integrated energy-momentum tensor and string proper size have been computed and analyzed.

The different methods to solve the string dynamics in curved apacetime have been characterized in terms of the parameter $c$ (or equivalently, in terms of the ratio between $\dot{X}^{A}$ and $\left.c X^{A^{\prime}}\right)$, as summarized in Sec. II and Table II:

(i) "Impulsive", $c \ll 1$ (null string expansion);

(ii) "Perturbative", $c=1$ (center of mass expansion);

(iii) "Adiabatic", $c \gg 1$ (dual to (i), initial configuration expansion);

(iv) "Composite", $(\tau \rightarrow 0$ expansion, ring string expansion).

The null string expansion is well appropriate to describe the string propagation in the strong gravitational field regime and well encompasses the $\tau \rightarrow 0$ expansion in cosmologiacal and black hole spacetimes, in the string unstable regime where the proper size of the string grows like the scalar factor, and like $\left(R_{S} / r\right)^{(D-3) / 2} \sim(-\tau)^{-(D-3) /(D+1)}$ inside the Schwarzschild black hole (near the $r=0$ singularity).

\section{ACKNOWLEDGMENTS}

C.O.L was supported by the NSF grant PHY-95-07719 and by research founds of the University of Utah. He also thanks the DEMIRM-Observatoire de Paris, where part of this work was done, for kind hospitality and use of its working facilities. 


\section{REFERENCES}

[1] M. B. Green, J. H. Schwarz and E. Witten, Superstring Theory, (Cambridge Univ. Press, Cambridge, 1987).

[2] N.D.Birrell and P.C.W.Davies, Quantum Field Theory in Curved Space (Cambridge U.P., Cambridge, 1982).

[3] H. J. de Vega and N. Sánchez in Proceedings of the Erice Course: "String Quantum Gravity and Physics at the Planck Energy Scale", 21-28 June 1992, edited by N. Sánchez, World Scientific, 1993.

[4] Proceedings of the Erice Course: "String Quantum Gravity and Physics at the Planck Energy Scale", 4-15 September 1995, edited by N. Sánchez and A. Zichichi, NATO ASI Series, C476, 1996.

[5] H.J.de Vega and N.Sánchez, Phys.Lett., B197, 320 (1987).

[6] M.Gasperini, N.Sánchez and G.Veneziano, Int.J.Mod.Phys., A6, 3853 (1991); Nucl. Phys. B364, 365 (1991).

[7] H.J.de Vega and A. Nicolaidis, Phys.Lett., B295, 214 (1992); H.J.de Vega, I.Giannakis and A. Nicolaidis, Modern Phys. Lett, A10, 2479 (1995).

[8] F. Combes, H. J. de Vega, A. V. Mikhailov and N. Sánchez, Phys. Rev. D50, 2754 (1994).

[9] H. J. de Vega, A. L. Larsen and N. Sánchez, Nucl. Phys., B427, 643 (1994).

[10] H. J. de Vega and I. L. Egusquiza, Phys. Rev. D49, 763 (1994).

[11] A. L. Larsen and N. Sánchez, Phys. Rev. D50, 7493 (1994).

[12] N. Sánchez and G. Veneziano, Nucl. Phys., B333, 253 (1990).

[13] C. O. Lousto and N. Sánchez, Phys. Rev. D47, 4498 (1993).

[14] H. J. de Vega and I. L. Egusquiza, preprint PAR-LPTHE/95-33, EHU-FT/9509, hepth/9506214.

[15] C. Lovelace, Phys.Lett., B135, 75 (1984).

[16] E. S. Fradkin and A. A. Tseylin, Nucl. Phys., B261, 1 (1985).

[17] A. L. Larsen, Phys. Rev. D50, 2623 (1994).

[18] A. L. Larsen and N. Sánchez, Phys. Rev. D51, 6929 (1995).

[19] H. J. de Vega, A. V. Mikhailov and N. Sánchez, Theor. Math. Phys., 94, 166 (1993).

[20] H. J. de Vega and N. Sánchez, Phys.Lett., B244, 215 (1990).

[21] H. J. de Vega and N. Sánchez, Phys. Rev. D42, 3969 (1990).

[22] H. J. de Vega and N. Sánchez, Int.J.Mod.Phys., A7, 3043 (1992).

[23] R. C. Myers and M. J. Perry, Ann. of Physics, Vol. 172, 304 (1986). 


\section{TABLES}

TABLE I. Here we recall the explicit conformal time $(\eta)$ and cosmic time $(t)$-dependence of the flat Friedmann-Robertson-Walker metric as usually referred to in the literature [6].

\begin{tabular}{ccl}
\hline \hline$R(\eta)=(\eta / \beta)^{\beta}$ & $R(t) \sim t^{\alpha=\beta /(\beta+1)}$ & Spacetime \\
\hline constant, $\beta \rightarrow 0$ & constant & Flat space \\
$\eta^{\beta}, 0<\beta<\infty$ & $t^{\alpha}, 0<\alpha<1$ & Standard cosmology \\
$e^{\eta}, \beta \rightarrow \infty$ & $t$ & Linear expansion \\
$\eta^{\beta},-1<\beta<0$ & $t^{\alpha},-\infty<\alpha<0$ & Super inflation \\
$\eta^{-1}$ & $e^{H t}$ & de Sitter \\
$\eta^{\beta},-\infty<\beta<-1$ & $t^{\alpha}, 1<\alpha<\infty$ & Power-law inflation \\
\hline \hline
\end{tabular}

TABLE II. In this table we show the different approaches to study the string dynamics in curved spacetimes. $c \ll 1, c=1$ and $c \gg 1$ correspond to $\partial_{\tau} X^{A} \gg c \partial_{\sigma} X^{A}, \partial_{\tau} X^{A} \sim c \partial_{\sigma} X^{A}$ and $\partial_{\tau} X^{A} \ll c \partial_{\sigma} X^{A}$ respectively. Hybrid cases are still possible when some components of the string coordinate $X^{A}$ satisfy eventually one of the inequalities and other coordinate components do not. The regimes of stability of the first order fluctuations are also shown as well as the references in which the approaches (in Cosmology and Black Holes) have been originally developped.

\begin{tabular}{|c|c|c|c|c|}
\hline \multirow[b]{2}{*}{ Approach } & \multicolumn{2}{|c|}{ Black Holes } & \multicolumn{2}{|c|}{ Cosmology } \\
\hline & Zeroth Order & $\begin{array}{c}\text { Fluctuations } \\
\qquad R \rightarrow 0\end{array}$ & Zeroth Order & $\begin{array}{l}\text { Fluctuations } \\
\qquad R \rightarrow \infty\end{array}$ \\
\hline $\begin{array}{c}\text { (i) Impulsive, } c \ll 1 \\
\dot{X}^{A} \gg c X^{A \prime}\end{array}$ & null string ${ }^{\mathrm{a}}$ & unstable & null string ${ }^{b}$ & unstable \\
\hline $\begin{array}{l}\text { (ii) Perturbative, } c=1 \\
\dot{X}^{A} \sim c X^{A \prime}\end{array}$ & center of mass ${ }^{\mathrm{c}}$ & unstable & center of mass ${ }^{\mathrm{d}}$ & stable/unstable \\
\hline $\begin{array}{l}\text { (iii) Adiabatic, } c \gg 1 \\
\dot{X}^{A} \ll c X^{A \prime}\end{array}$ & initial configuration $^{a}$ & unstable & initial configuration $^{\mathrm{a}}$ & unstable \\
\hline (iv) Hybrid & $\operatorname{ring}^{\mathrm{e}}$ & unstable & asymptotic ${ }^{\mathrm{f}}$ & stable/unstable \\
\hline
\end{tabular}

aThis paper

${ }^{\mathrm{b}}$ Ref. [7].

${ }^{\mathrm{c}}$ Ref. 13.

${ }^{\mathrm{d}}$ Ref. [5].

${ }^{\mathrm{e}}$ Ref. 17.

${ }^{\mathrm{f}}$ Ref. [6]. 